\title{
Neue Impulse der Europäischen Kommission für eine europäische Weltraumstrategie
}

\author{
Markus Hesse und Marcus Hornung*
}

\section{Die europäische Weltraumpolitik - ein Politikfeld sui generis}

Seit dem Beschluss, das europäische Satellitennavigationssystem Galileo aus Mitteln des Haushalts der Europäischen Union zu finanzieren, ${ }^{1}$ wurde dieses Flaggschiffprojekt der europäischen Weltraumpolitik (EWP) zunehmend aus der ,Schussbahn' der politischen Debatten genommen. Zudem ist inzwischen die mediale Aufmerksamkeit hinsichtlich der EWP merklich zurückgegangen. Besonders der Vertrag von Lissabon von 2009 und die Mitteilung der Kommission „Auf dem Weg zu einer Weltraumstrategie der Europäischen Union im Dienst der Bürgerinnen und Bürger" ${ }^{“ 2}$ von 2011 sowie die Verhandlungen über den mehrjährigen Finanzrahmen von 2014 bis 2020 geben jedoch Anlässe, sich gerade jetzt des Themenfeldes anzunehmen. Der im Zuge des Vertrags von Lissabon neu aufgenommene Art. 189 Vertrag über die Arbeitsweise der Europäischen Union (AEUV) verpflichtet die Europäische Union, eine ,zweckdienliche Verbindung “3 zur Europäischen Weltraumorganisation (ESA) herzustellen. Jedoch führt die unterschiedliche Auslegung einer „zweckdienlichen Verbindung“ ebenso wie ihre konkrete Umsetzung zu einigen Problemen, was sich etwa bei der Realisierung von Galileo mehrfach deutlich zeigt.

Die EWP ist nicht nur als ein Politikfeld sui generis zu bezeichnen, da mit der Europäischen Union und der ESA zwei bedeutende internationale Akteure gemeinsam auf europäischer Ebene agieren. Des Weiteren ist Weltraumpolitik generell ein einzigartiges Querschnittsthemenfeld, das enge Bezüge zu Forschungs- und Technologiepolitik, Sicherheitsund Verteidigungspolitik, Wirtschafts- und Finanzpolitik sowie weiteren Politiken aufweist. Der Weltraum bietet, allein schon aufgrund seiner einzigartigen physikalischen Eigenschaften, unzählige Nutzungsmöglichkeiten. Das amerikanische Satellitennavigationssystem Global Positioning System (GPS) ist das bekannteste Beispiel und wird so intensiv unter anderem

* Dr. Markus Hesse, M.A., M.E.S., Projektleiter, Büro des Rektors, Technische Universität Chemnitz.

Marcus Hornung, M.E.S., Wissenschaftlicher Mitarbeiter, Jean-Monnet-Professur für Europäische Integration, Technische Universität Chemnitz.

1 Vgl. Europäische Kommission: Mitteilung der Kommission an das Europäische Parlament, den Rat, den Europäischen Wirtschafts- und Sozialausschuss und den Ausschuss der Regionen. Galileo am Scheideweg: Die Umsetzung der Europäischen GNSS-Programme, KOM (2007) 261; Rat der Europäischen Union: Mitteilung an die Presse: 2833. Tagung des Rates Wirtschaft und Finanzen, 15231/07; Verordnung (EG) Nr. 683/2008 des Europäischen Parlaments und des Rates vom 9. Juli 2008 über die weitere Durchführung der europäischen Satellitennavigationsprogramme (EGNOS und Galileo), in: Amtsblatt der EU, Nr. L 196 vom 24. Juli 2008, S. 1-11.

2 Europäische Kommission: Mitteilung der Kommission an das Europäische Parlament, den Rat, den Europäischen Wirtschafts- und Sozialausschuss und den Ausschuss der Regionen. Auf dem Weg zu einer Weltraumstrategie der Europäischen Union im Dienst der Bürgerinnen und Bürger, KOM (2011) 152; Vgl. hierzu bisher lediglich Marcel Dickow: Die Weltraumpolitik der EU. Zivile Flaggschiffe und Optionen für die GSVP, Stiftung Wissenschaft und Politik: SWP-Studie, Berlin 2011, S. 19; Benedikt Langner/Marcus Schwenke: EU-Weltraumstrategie, Centrum für Europäische Politik: CEP Kurzanalyse, Freiburg 2011.

3 Art. 189 Abs. 3 AEUV; vgl. Henning Eikenberg: Art. 189 AEUV, in: Eberhard Grabitz/Meinhard Hilf/Martin Nettesheim (Hrsg.): Das Recht der Europäischen Union, München 2011, Art. 189 AEUV, Rn. 32-40; Hans-Heinrich Trute/Arne Pilniok: Art. 189 AEUV, in: Rudolf Streinz (Hrsg.): EUV/AEUV, Vertrag über die Europäische Union und Vertrag über die Arbeitsweise der Europäischen Union, München 2012, Art. 189 AEUV, Rn. 6. 
in militärischen, verkehrspolitischen und privaten Bereichen genutzt, dass es kaum mehr wegzudenken ist. Aber die gegenwärtig operativen Satellitensysteme offerieren den Nutzern weit mehr: Beispielsweise bieten Erdbeobachtungsprogramme ein breites Spektrum an Fähigkeiten, wie die Bereitstellung von Umwelt- und Wetterdaten, die Rückschlüsse auf Naturkatastrophen oder den Klimawandel zulassen. Neben meteorologischen können ebenso geophysikalische Gefahren früher erkannt werden, was beim Katastrophenmanagement eine herausragende Unterstützung darstellt. Satellitenkommunikation ist ein weiterer dieser Anwendungsbereiche, der zunehmend an Bedeutung gewinnt.

Die zentralen Themenbereiche Finanzen und Sicherheit sorgen auf internationaler Ebene für eine besondere Brisanz bei Verhandlungen über neue Weltraumanwendungen, Änderungen des Weltraumrechts oder der Zuweisung von Frequenzen und Orbits. ${ }^{4}$ Die Themen stellen damit auch einen Sonderfall der Kooperationsbeziehungen der Europäischen Union und der ESA dar. Auch die Mitgliederstruktur beider Organisationen ist ähnlich, aber eben nicht deckungsgleich: 17 der 19 ESA-Mitgliedstaaten sind gleichzeitig EU-Mitglieder folglich sind längst nicht alle EU-Mitgliedstaaten auch ESA-Mitglieder, während die Schweiz und Norwegen zwar in der ESA, aber nicht Teil der Europäischen Union sind. Die ESA ist zudem eine rechtlich von der Europäischen Union vollkommen unabhängige Organisation. Unterschiede in Ausrichtung und Funktionsweisen gelten als Hinweise dafür, dass die Zusammenarbeit mit der ESA als Beispiel der EU-Außenvertretung gegenüber Dritten behandelt werden muss. Die Europäische Kommission strebt in dieser Situation eine aktivere Rolle der Union in der EWP an. Sie begründet dies mit einem, ,ausdrücklichen Mandat ${ }^{\text {“5 }}$ der Europäischen Union auf der Grundlage von Art. 189 AEUV, nach dem die Weltraumpolitik nunmehr eine geteilte Zuständigkeit der Union und der Mitgliedstaaten ist. Des Weiteren erklärt sie, dass die politische Dimension und die Koordinationsaufgaben zu anderen Politikbereichen eine verstärkte Rolle der Europäischen Union - und damit natürlich auch der Kommission selbst - erforderlich machen. ${ }^{6}$ Diese verschiedenen Auffassungen führten seit Beginn des Engagements der Europäischen Union in der Weltraumpolitik zu Komplikationen im Verhältnis zwischen Europäischer Union und ESA.

Weltraumpolitik wird grundsätzlich auf drei Ebenen betrachtet: ${ }^{7}$ Ihren Ursprung hat die moderne Raumfahrt auf nationaler Ebene in den USA sowie der damaligen Sowjetunion. Politisch motiviert standen sich beide Blöcke in der Phase des „Space 1.0“8 im Sinne einer bipolaren Struktur und eines ,Wettlaufs ins All` gegenüber. Daraufhin ergab sich im Laufe der 1960er Jahre ein Regulierungsbedarf, dem auf der zweiten, der international-völkerrechtlichen Ebene und somit auf Ebene der Vereinten Nationen begegnet wurde. Der Welt-

4 Vgl. exemplarisch die diplomatischen Auseinandersetzungen zwischen der Europäischen Union und China um die Frequenzen des Galileo-Systems: Yan Liang: China to launch 2 satellites for Compass navigation system, in: China Daily, 13.11.2006; Taylor Dinerman: China and Galileo continued, in: The Space Review, 21.8.2006; Geoffrey Forden: The Military Capabilities and Implications of China's Indigenous Satellite-Based Navigation System, in: Science and Global Security 3/2004, S. 219-250; Shu-Hsien Liao: Will China become a military space superpower?, in: Space Policy 3/2005, S. 205-212.

5 Europäische Kommission: Auf dem Weg zu einer Weltraumstrategie, 2011, S. 3.

6 Vgl. ebenda, S. 13.

7 Vgl. Wulf von Kries/Bernhard Schmidt-Tedd/Kai-Uwe Schrogl: Grundzüge des Raumfahrtrechts. Rahmenbestimmungen und Anwendungsgebiete, München 2002, S. 4.

8 Nicolas Peter: Space power and its implications - The case of Europe, in: Acta Astronautica 3-4/2010, S. 348-354, hier S. 350 . 
raumvertrag von 1967 - die „Magna Charta des Weltraumrechts“9 - und seine Folge- und Ergänzungsverträge bilden noch immer die Grundlage im besonderen Völkerrecht. Bis heute ist das „United Nations Commitee on the Peaceful Uses of Outer Space“ (UNCOPUOS) mitsamt seinen Unterausschüssen das Diskussionsforum weltraumrechtlicher und -politischer Fragen auf internationalem Parkett. ${ }^{10}$ Zwischen die nationale und völkerrechtlich-internationale gliedert sich die dritte, die europäische Ebene, die im Fokus des vorliegenden Artikels steht, als jüngste der drei genannten ein. Ihre Aufgabe besteht darin, die in den europäischen Staaten existenten Weltraumbestrebungen zu koordinieren und zugleich integrierte Weltraumprogramme durchzuführen. ${ }^{11}$

Die getrennt zu betrachtenden Hauptakteure der EWP sind die Europäische Union und die ESA. ${ }^{12}$ Ihre weltraumpolitischen Motivationen unterscheiden sich maßgeblich:

Die ESA wurde als internationale Organisation mit Sitz in Paris und damals zehn Mitgliedern im Jahr 1975 gegründet. ${ }^{13}$ Die ESA-Konvention ist zugleich Gründungsdokument und gegenwärtige Handlungsgrundlage. Gemäß dieser dient die Organisation der Zusammenarbeit in den Bereichen Weltraumforschung und -technologie, ${ }^{14}$ da Raumfahrt die personellen, technischen und finanziellen Möglichkeiten der einzelnen europäischen Staaten überfordert. ${ }^{15}$ Kurzum zielt die ESA auf die Optimierung der wirtschaftlichen und technologischen Aspekte der Raumfahrt ab. Jedoch tritt sie längst nicht mehr nur als derartige Weltraumagentur, sondern auch als Akteur der EWP auf, wie nicht zuletzt die zahlreichen politischen Implikationen der strategieweisenden „ESA-Agenden“16 belegen. Explizit betont die Europäische Kommission, dass ihre Mitteilung „Europäische Raumfahrtpolitik“ zum einen in Absprache mit den Mitgliedstaaten beider Organisationen entstanden ist und zum anderen erstmals ein gemeinsames Dokument von Europäischer Kommission und ESA-Generaldirektor darstellt. ${ }^{17}$

Die Europäische Union widmet sich erst seit den 1990er Jahren dem Themenfeld Weltraum und entwickelte sich seit dieser Zeit zu einem aufstrebenden und zunehmend ambitionierten weltraumpolitischen Akteur. Anders als für die ESA ist die Anwendung von Weltraumprojekten für die Europäische Union seit circa zehn Jahren ein strategisches politisches Interesse. ${ }^{18}$ Weltraumpolitik ist für die Europäische Union als Politikfeld zu etablieren, um „ein besserer Nachbar und ein respektierter Partner der globalen Gemeinschaft zu sein“19 und um „dazu bei[zu]tragen, das politische Ansehen der Europäischen Union in der Welt zu

9 Marcel Dickow: The European Union proposal for a Code of Conduct on Outer Space Activities, in: Kai-Uwe Schrogl/Charlotte Mathieu/Nicolas Peter (Hrsg.): Yearbook on Space Policy 2007/2008, Wien 2009, S. 152-163, hier S. 155; von Kries/Schmidt-Tedd/Schrogl: Grundzüge des Raumfahrtrechts, 2002, S. 7.

10 Vgl. Gérard Brachet: Peace in Space: Building on the Outer Space Treaty, in: United Nations Institute for Disarmament Research (Hrsg.): 50 Years of Space Technology, 40 Years of the Outer Space Treaty, Genf 2007, S. 67-76, hier S. 67; Wolfgang Rathgeber/Nina-Louisa Remuss: Space Security, A Formative Role and Principled Identity for Europe, European Space Policy Institute: ESPI Report, Wien 2009, S. 11-12; von Kries/ Schmidt-Tedd/Schrogl: Grundzüge des Raumfahrtrechts, 2002, S. 7.

11 Vgl. Europäische Kommission: Mitteilung der Kommission an den Rat und das Europäische Parlament: Europäische Raumfahrtpolitik, KOM (2007) 212, S. 5 .

12 Vgl. Rathgeber/Remuss: Space Security, 2009, S. 35.

13 Vgl. Stephan Hobe/Katharina Kunzmann/Thomas Reuter: Rechtliche Rahmenbedingungen einer zukünftigen kohärenten Struktur der europäischen Raumfahrt, Berlin 2006, S. 5.

14 Vgl. Art. 2 ESA-Konvention.

15 Vgl. Präambel der ESA-Konvention.

16 Vgl. European Space Agency: Agenda 2007, Paris 2003; European Space Agency: Agenda 2011, Paris 2007.

17 Vgl. Europäische Kommission: Europäische Raumfahrtpolitik, 2007, S. 4.

18 Ebenda, S. 4.

19 Vgl. Europäische Kommission: Weißbuch. Die Raumfahrt: Europäische Horizonte einer erweiterten Union, Aktionsplan für die Durchführung der europäischen Raumfahrtpolitik, KOM (2003) 673, S. 8-9. 
mehren, ihre wirtschaftliche Wettbewerbsfähigkeit zu stärken und ihre Reputation im Bereich wissenschaftlicher Höchstleistungen auszubauen. "20 Zudem hat die Kommission mehrfach geäußert, dass Weltraumpolitik eine Querschnittspolitik darstellt, die zahlreiche Felder des alltäglichen Lebens betrifft. ${ }^{21}$ Nicht zuletzt verfolgt die EU-Weltraumpolitik nach außen die ambitionierte und komplexe Zielsetzung, Wissenschaft und Technologie mit Außenpolitik zu verknüpfen. Mittlerweile wird gar von einer „Weltraumdiplomatie“,22 zum Beispiel gegenüber anderen Weltraummächten, wie den USA, Russland und China, gesprochen.

ESA und Europäische Union verfolgen demnach unterschiedliche weltraumpolitische Ziele. Dabei ergänzen sie sich, sodass eine Kooperation im Lauf der Jahrzehnte von den beteiligten Akteuren als sinnvoll erachtet wurde. Allein aus finanziellen Gründen erscheint eine Zusammenarbeit vernünftig, zumal Weltraumprojekte generell mit hohen Kosten verbunden sind. Daraus resultieren allerdings Konflikte, die eine Zusammenarbeit erschweren: Erstens sollten Kosten aufgrund der Kooperation gespart und nicht durch eine teure Verdopplung von Organisationsstrukturen und eine unnötige Konkurrenz in die Höhe getrieben werden. Zentrale Fragen sind hier: Wer übernimmt welche Aufgaben bei der Zusammenarbeit? Wer ist Auftraggeber? Wer setzt die Projekte um? Wer bezahlt? Zweitens müssten sich die beiden Organisationen auf gemeinsame Projekte einigen: Die zentrale Frage hier ist, ob sich die ESA dem bisher vermiedenen Feld der Sicherheits- und Verteidigungspolitik zuwendet, um auch dort die Wünsche der Europäischen Union nach mehr Weltraumanwendungen für eine bedeutendere Rolle in der Außen- und Sicherheitspolitik zu erfüllen. Daraus ergeben sich zwangsläufig weitere rechtliche und politische Fragen, die zu diskutieren sind.

\section{Auf dem Weg zu einer tragfähigen Kooperationsbasis zwischen EU und ESA}

\section{Die Annäherung zwischen Europäischer Union und ESA seit 2000}

Seit dem Jahr 2000 näherten sich die Europäische Union und die ESA einer institutionalisierten Kooperation an. Vier ausgewählte Meilensteine sollen diesen Weg nachzeichnen und diese Einschätzung begründen:

Mithilfe eines Grün- und Weißbuchprozesses ${ }^{23}$ näherte sich die Europäische Kommission den Anforderungen an eine kohärente EWP. Das Weißbuch enthält ein zweistufiges Konzept der Annäherung an die ESA: Bis 2007 sollten die Europäische Union und die Europäische Weltraumorganisation auf der Grundlage eines Rahmenabkommens ${ }^{24}$ (EU-ESA-RA) agieren, wobei die Aufgabenteilung deutlich wird: „Die ESA sollte die Durchführungsstelle der Union in Raumfahrtangelegenheiten sein. “25 Dann hätte gemäß der damaligen Planung der Vertrag über eine Verfassung für Europa in Kraft treten sollen, der die ESA in einer zweiten Phase ab 2007 nach den im Weißbuch erklärten Vorstellungen stärker in den Rahmen der Europäischen Union eingebunden hätte. Zudem wäre die Raumfahrtpolitik auch bei Inkraft-

20 Ebenda, S. 9.

21 Vgl. Europäische Kommission: Europäische Raumfahrtpolitik, 2007, S. 4.

22 Nicolas Peter: The EU's emergent space diplomacy, in: Space Policy 2/2007, S. 97-107, hier S. 97.

23 Europäische Kommission: Grünbuch. Europäische Raumfahrtpolitik, KOM (2003) 17; Europäische Kommission: Weißbuch. Aktionsplan für die Durchführung der europäischen Raumfahrtpolitik, 2003.

24 Rahmenabkommen zwischen der Europäischen Gemeinschaft und der Europäischen Weltraumorganisation, in: Amtsblatt der EU, Nr. L 261 vom 6. August 2004, S. 64-84.

25 Europäische Kommission: Weißbuch. Aktionsplan für die Durchführung der europäischen Raumfahrtpolitik, 2003, S. 43. 
treten des Verfassungsvertrags als ein Politikbereich gemeinsamer Zuständigkeit der Europäischen Union und ihrer Mitgliedstaaten definiert worden. ${ }^{26}$

Wenige Tage nach Veröffentlichung des Weißbuchs wurde am 25. November 2003 das EU-ESA-RA unterzeichnet, welches 2004 in Kraft trat. Das EU-ESA-RA ist ein klassischer völkerrechtlicher Vertrag und legt die rechtliche Kooperationsbasis zwischen Europäischer Union und ESA dar. Seine Laufzeit betrug vorerst vier Jahre, eine weitere Verlängerung ergibt sich automatisch bei Nicht-Kündigung von einer der beiden Seiten.

2007 erfolgte die Verabschiedung der EWP durch eine Entschließung des Rates, ${ }^{27}$ der eine Kommissionsmitteilung ${ }^{28}$ vorausgegangen war. Entgegen positiveren Erwartungen wurden keine Veränderungen an den vorhandenen Kooperationsmechanismen vorgenommen. In der Kommissionsmitteilung heißt es jedoch: „Das EG-ESA-Rahmenabkommen liefert solide Grundvoraussetzungen für die Koordinierung von zwischenstaatlichen Maßnahmen und Maßnahmen der Gemeinschaft. “29 Das EU-ESA-RA blieb Kooperationsgrundlage, hinsichtlich seiner Effizienz wurden jedoch Verbesserungen angeregt.

Die im Weißbuch 2003 angekündigte zweite Kooperationsphase konnte durch das Scheitern des Verfassungsvertrags bis dato nicht eingeleitet werden. Allerdings nimmt der 2009 in Kraft getretene Vertrag von Lissabon erstmals Weltraumpolitik als zwischen Europäischer Union und ihren Mitgliedstaaten geteilte Kompetenz ${ }^{30}$ in das Primärrecht der Europäischen Union auf. Hinsichtlich der Kooperationsmechanismen zwischen Europäischer Union und ESA verpflichtet Art. 189 Abs. 3 AEUV die Europäische Union, „Zweckdienliche Verbindungen“" zur ESA herzustellen. ${ }^{31}$ Er selbst nimmt keine Änderungen am Kooperationsfundament des EU-ESA-RA vor. ${ }^{32}$

Resultat dieser Entwicklung ist, dass das EU-ESA-RA bis heute die gültige Kooperationsgrundlage zwischen Europäischer Union und ESA darstellt, ${ }^{33}$ da es die identifizierten Meilensteine unangetastet lässt.

\section{Das EU-ESA-Rahmenabkommen als Kooperationsbasis seit 2004}

Warum eine Überarbeitung des Status quo erforderlich ist, stellt die folgende Betrachtung des EU-ESA-RA dar. Um die wesentlichen Bestimmungen des Kooperationsabkommens zu verstehen, sind die einschlägigen Artikel zu beleuchten:

Art. 1 und 2 EU-ESA-RA arbeiten die gemeinsamen weltraumpolitischen Ziele von Europäischer Union und ESA und darauf aufbauend Grundlagen der Zusammenarbeit heraus. Die Vertragsparteien kommen überein, dass das Abkommen die Absicht verfolgt, eine ,[k]ohärente und schrittweise Entwicklung einer umfassenden europäischen Raumfahrtpo-

26 Vgl. Europäische Kommission: Weißbuch. Aktionsplan für die Durchführung der europäischen Raumfahrtpolitik, 2003, S. 43.

27 Rat der Europäischen Union: Entschließung vom 21. Mai 2007 zur Europäischen Raumfahrtpolitik, in: Amtsblatt der EU, Nr. C 136 vom 20. Juni 2007, S. 1-5.

28 Europäische Kommission: Europäische Raumfahrtpolitik, 2007.

29 Ebenda, S. 14.

30 Vgl. Eikenberg: Art. 189 AEUV, 2011, Rn. 9; Kotzur: Art. 189 AEUV, 2010, Rn. 2; Mönig: Art. 189 AEUV, 2010, Rn. 7.

31 Vgl. Eikenberg: Art. 189 AEUV, 2011, Rn. 32-40; Fischer: Text und Kommentar zum Europäischen Reformvertrag, 2010, Art. 189 AEUV; Kotzur: Art. 189 AEUV, 2010, Rn. 7; Mönig: Art. 189 AEUV, 2010, Rn. 5, 11; Ruffert: Art. 189 AEUV, 2011, Rn. 4-5; Trute/Pilniok: Art. 189 AEUV, 2012, Rn. 6.

32 Vgl. Jan Wouters: Space in the Treaty of Lisbon, in: Kai-Uwe Schrogl/Charlotte Mathieu/Nicolas Peter (Hrsg.): Yearbook on Space Policy 2007/2008, Wien 2009, S. 116-124, hier S. 122.

33 Vgl. Dickow: Die Weltraumpolitik der EU, 2011, S. 8. 
litik“"34 voranzutreiben. Konkrete Mechanismen dazu werden noch nicht benannt. Art. 3 EUESA-RA formuliert als Kooperationsbereiche zwischen Europäischer Union und ESA, auf die die Instrumentarien des Abkommens anwendbar sein werden: Wissenschaft, Technologie, Satellitenkommunikation, Mikrogravitation, Erdbeobachtung, Schiffsführung, bemannte Raumfahrt, Trägerraketen und weltraumbezogene Frequenzpolitik. ${ }^{35}$ Auch wenn die Liste der Kooperationsbereiche nicht abschließend ist, ${ }^{36}$ fällt doch auf, dass einige weltraumpolitische Kernfragen nicht Teil des EU-ESA-RA sind. Es handelt sich dabei zum Beispiel um die Industriepolitik und die sicherheitspolitische Nutzung europäischer Weltraumanwendungen. ${ }^{37}$ Art. 4 und 5 EU-ESA-RA regeln als Herzstück des Übereinkommens die Umsetzung der genannten Ziele und die Ausgestaltung der Kooperationsbereiche. Dies geschieht mithilfe vorformulierter „Initiativen“, 38 die das jeweilige programmbezogene Aufgabengefüge charakterisieren: So ist es denkbar, dass a) die ESA als Managementorganisation der EU-Weltraumprojekte auftritt, b) die Europäische Union an bestehenden Programmen der ESA mitwirkt, c) gemeinsam koordinierte und finanzierte Projekte durchgeführt werden, d) Dritte für die Europäische Union und die ESA Maßnahmen ergreifen oder e) sonstige gemeinsame Untersuchungen, Symposien, Workshops usw. durchgeführt werden. Das EUESA-RA trifft damit keine Aussagen über klare EWP-Zuständigkeitsabgrenzungen. Vielmehr kommen die Vertragsparteien überein, gegebenenfalls nach Art. 4 Abs. 2 EU-ESA-RA mit Sondervereinbarungen die Regulierungslücken zu schließen. Art. 6 EU-ESA-RA sieht Regeln zur Information und Konsultation zwischen Europäischer Union und ESA vor, die sich nach Art. 7 EU-ESA-RA auch auf die jeweilige weltraumpolitische Außenwirkung gegenüber Dritten, also beispielsweise anderen Weltraummächten, der beiden Organisationen erstrecken. Art. 8 EU-ESA-RA schafft durch das Einsetzen des Weltraumrats eine scheinbar neue institutionelle Struktur der EWP. Der Weltraumrat ist ein regelmäßig tagendes Gremium auf Ministerebene, ${ }^{39}$ der jedoch über keinerlei Entscheidungsbefugnis verfügt. Er kann insofern eher als ,gleichzeitige Tagung der Räte von EU und ESA“40 angesehen werden, die dem Rat der Europäischen Union und der ESA nicht übergeordnet sind. Daher müssen seine Entscheidungen von beiden Räten grundsätzlich nach deren jeweiligen Verfahren angenommen werden. Der Weltraumrat wird durch ein Sekretariat und eine High Level Space Policy Group $^{41}$ unterstützt.

Diese Bestimmungen überblickend muss eine zweigeteilte Bewertung der Kooperationsgrundlage EU-ESA-RA vorgenommen werden. Zum einen liegt die Errungenschaft des Vertrags als erste formalisierte Zusammenarbeit vor allem darin, eine ständige Kommunikationsplattform zur Abstimmung der jeweiligen Weltraumpolitiken geschaffen zu haben. ${ }^{42}$ Damit wurden Instrumentarien, die schon zuvor genutzt wurden und damit nicht als

34 Art. 1 Abs. 1 EU-ESA-RA.

35 Vgl. Art. 3 Abs. 1 EU-ESA-RA.

36 Vgl. Art. 3 Abs. 2 EU-ESA-RA.

37 Vgl. Hobe/Kunzmann/Reuter: Rechtliche Rahmenbedingungen, 2006, S. 244.

38 Art. 4 Abs. 1 EU-ESA-RA.

39 Vgl. Art. 8 Abs. 1 EU-ESA-RA.

40 Hobe/Kunzmann/Reuter: Rechtliche Rahmenbedingungen, 2006, S. 245.

41 Vgl. Art. 8 Abs. 3-4 EU-ESA-RA.

42 Vgl. Stephan Hobe/Oliver Heinrich/Irina Kerner/Annette Froehlich: Entwicklung der Europäischen Weltraumagentur als ,,implementing agency“ der Europäischen Union: Rechtsrahmen und Anpassungserfordernisse, Berlin 2009, S. 12, S. 304. 
innovativ zu bezeichnen sind, in verbindliches Recht gegossen. ${ }^{43}$ Das ist insofern zwar keine Neuerung, bietet aber zumindest einen kohärenten rechtlichen Rahmen für die Zusammenarbeit der beiden Vertragsparteien. Zum anderen hat sich aber als problematisch herausgestellt, dass die geschaffene Institution Weltraumrat ohne echte Entscheidungsbefugnisse nur begrenzten Wert entfaltet. Erst in jüngster Zeit nimmt der Weltraumrat verstärkten Einfluss auf die Entwicklung einer EWP. ${ }^{44}$ Für die in Art. 1 Abs. 1 EU-ESA-RA manifestierte Zielstellung einer kohärenten und damit effektiveren EWP werden allerdings kaum nennenswerte Fortschritte erzielt. ${ }^{45}$ Weiterhin wird keine deutliche Kompetenz- und Aufgabenabgrenzung zwischen Europäischer Union und ESA vorgenommen, sodass das Abkommen letztlich nur zwei abstrakte Kooperationsformen als realistisch auf Projekte anwendbar vorschlägt: Dabei handelt es sich um die Ad-hoc-Kooperationen, die nach Art. 4 Abs. 2 EUESA-RA einzeln abzustimmende Sondervereinbarungen notwendig machen, sowie um die Managementoption. ${ }^{46}$ Beide Kooperationsformen setzten doppelte und individuelle Entscheidungsfindungsmechanismen voraus. Die im Abkommen erfassten Initiativen stellen nur den kleinsten gemeinsamen Nenner europäischer Weltraumkooperation dar.

Das EU-ESA-RA stellt somit zwar einen Fortschritt für die EWP dar, ist aber für einige der wichtigsten Bereiche aktueller und zukünftiger EWP, wie Anwendungen im Rahmen der Gemeinsamen Sicherheits- und Verteidigungspolitik (GSVP), eine unzureichende Kooperationsgrundlage. Daraus resultiert Handlungsbedarf hinsichtlich einer überarbeiteten künftigen Kooperationsbasis, den auch die Europäische Kommission mittlerweile erkannt hat. Sie hat daher vorgeschlagen, die Kooperation zwischen ESA und Europäischer Union auf eine neue Grundlage zu stellen. ${ }^{47}$

\section{Finanzen und Sicherheit als Problemfelder einer gemeinsamen europäischen Weltraumpolitik}

Die bisherigen Ausführungen haben zum einen den aktuellen Stand der EU-ESA-Kooperation, zum anderen Problembereiche in dieser Zusammenarbeit dargelegt. Nach welchen komplementären Prämissen die Europäische Union und die ESA in den betroffenen Politikfeldern agieren, soll hier an zwei Beispielen - aus den Themenfeldern Finanzen und Sicherheit - veranschaulicht werden.

Ein erstes Beispiel eines EU-ESA-Konfliktfeldes ist industriepolitischer Natur: Sowohl die Europäische Union als auch die ESA müssen Aufträge an die Weltraumindustrie vergeben. Da die Raumfahrt eine Schlüsselbranche mit erheblichem Forschungsaufwand ist, sind die entsprechenden Aufträge für die einschlägigen Unternehmen, ihre Standorte und betroffenen Staaten von größter Bedeutung. Die vergaberechtlichen Grundlagen beider Organisationen differieren allerdings elementar. ${ }^{48}$

43 Vgl. Thomas Reuter: The framework agreement between the European Space Agency and the European Community: A significant step forward?, in: Zeitschrift für Luft- und Weltraumrecht 1/2004, S. 56-65, hier S. 65.

44 Vgl. Alain Gaubert/André Lebeau: Reforming European space governance, in: Space Policy 1/2009, S. 37-44, hier vor allem S. 43.

45 Vgl. Hobe/Kunzmann/Reuter: Rechtliche Rahmenbedingungen, 2006, S. 239.

46 Vgl. Hobe/Heinrich/Kerner/Froehlich: Entwicklung der Europäischen Weltraumagentur, 2009, S. 12, S. 304.

47 Vgl. Europäische Kommission: Auf dem Weg zu einer Weltraumstrategie, 2011, S. 14.

48 Vgl. Dickow: Die Weltraumpolitik der EU, 2011, S. 9-10. 
Das Vergaberecht der Europäischen Union basiert auf den vier Grundfreiheiten und wird sekundärrechtlich durch die Vergabekoordinierungs-49 und die Sektorenkoordinierungsrichtlinie ${ }^{50}$ ausgestaltet. Die wesentlichen Kriterien zur Auftragsvergabe werden in der Vergabekoordinierungsrichtlinie zusammengefasst: „Die Zuschlagserteilung sollte auf der Grundlage objektiver Kriterien erfolgen, die [...] sicherstellen, dass die Angebote unter wirksamen Wettbewerbsbedingungen bewertet werden. Dementsprechend sind nur zwei Zuschlagskriterien zuzulassen: das des ,niedrigsten Preises ${ }^{6}$ und das des ,wirtschaftlich günstigsten Angebots““. 51

Die ESA-Vergabegrundlage bildet die industriepolitische Anlage V zur ESA-Konvention. Sie legt die vergaberechtliche Besonderheit fest, auf der sämtliche ESA-Projekte basieren: die geografische Verteilung. Das sogenannte Rückflussprinzip besagt, dass nach einem Rückflusskoeffizienten die durch einen ESA-Mitgliedstaat eingebrachten Finanzmittel in Form von Aufträgen an die jeweilige Weltraumindustrie dieses Mitgliedstaats zurückfließen. Der Rückflusskoeffizient darf nicht unter 0,8 liegen, ${ }^{52}$ ist in der Praxis sogar meist bei 0,9 oder höher. ${ }^{53}$ Das bedeutet, dass mindestens 80 Prozent der gezeichneten Finanzmittel an die Weltraumindustrie in dem jeweiligen Land zurückfließen müssen. Somit ist im Idealfall ein reges Engagement der Mitgliedstaaten sichergestellt und zugleich wird die heimische Industrie- und Forschungslandschaft gestärkt.

Unschwer zu erkennen ist, dass sich dieses Prinzip nicht mit den vergaberechtlichen Grundsätzen der Europäischen Union vereinbaren lässt, ${ }^{54}$ die auf Nichtdiskriminierung und Wirtschaftlichkeit basieren. Sobald die Europäische Union und die ESA Kooperationsprojekte unterhalten, führt dies zwangsläufig zum Konflikt, den das EU-ESA-RA dadurch ausräumt, dass in gemeinsamen Unternehmungen allein das Vergaberecht der Europäischen Union angewendet wird und das ESA-Rückflussprinzip nicht gilt. ${ }^{55}$ Dies ist pragmatisch, obgleich es das grundsätzliche Problem nicht löst: So sind aber praktisch nur Kooperationsszenarien denkbar, in denen entweder anders lautende Vereinbarungen getroffen werden oder die ESA als Durchführungsagentur der Europäischen Union fungiert.

Als zweites Beispiel eines EU-ESA-Konflikts dient der Themenbereich Sicherheit, der in diesem Zusammenhang nicht nur rein militärisch, sondern auch mit Bezug auf andere Poli-

49 Richtlinie 2004/18/EG des Europäischen Parlaments und des Rates vom 31. März 2004 über die Koordinierung der Verfahren zur Vergabe öffentlicher Bauaufträge, Lieferaufträge und Dienstleistungsaufträge, in: Amtsblatt der EU, Nr. L 134 vom 30. April 2004, S. 114-240.

50 Richtlinie 2004/17/EG des Europäischen Parlaments und des Rates vom 31. März 2004 zur Koordinierung der Zuschlagserteilung durch Auftraggeber im Bereich der Wasser-, Energie- und Verkehrsversorgung sowie der Postdienste, in: Amtsblatt der EU, Nr. L 134 vom 30. April 2004, S. 1-113.

51 Erwägung 46 Richtlinie zur Vergabe öffentlicher Bauaufträge, Lieferaufträge und Dienstleistungsaufträge.

52 Vgl. ESA-Konvention Anl. 5 Art. 4 Abs. 6.

53 Vgl. Kevin Madders: A New Force at a New Frontier. Europe's development in the space field in the light of its main actors, policies, law and activities from its beginnings up to the present, Cambridge 1997, S. 395-397; Hobe/Kunzmann/Reuter: Rechtliche Rahmenbedingungen, 2006, S. 375-381. Zur Auftragsvergabe, Beschaffungspolitik und möglichen Überarbeitung der ESA-Vergaberegeln vgl. auch: Hobe/Heinrich/Kerner/Froehlich: Entwicklung der Europäischen Weltraumagentur, 2009, S. 200-208. Zu den ersten Verhandlungen über eine Flexibilisierung der Rückflussregel vgl. Eros Pittarelli: ESA's Industrial Policy after the FINPOL Initiative, in: esa bulletin 120/2004, S. 54-55. Die Diskussion über neue ESA-Vergaberegeln wurde auf einem internationalen Symposium der ESA im Mai 2007 fortgesetzt, blieb jedoch ohne Ergebnis, vgl. dazu: Isabelle Arnold/Christian Plingen: International Symposium: Developing Trends in Public Procurement and Auditing, Noordwijk, the Netherlands, May, 14 to 16, 2007, in: Zeitschrift- für Luft- und Weltraumrecht 4/2007, S. 627-629, hier S. 627-628.

54 Vgl. Gaubert/Lebeau: Reforming European space governance, 2009, S. 43.

55 Vgl. Art. 5 Abs. 3 EU-ESA-RA. 
tikbereiche und damit umfassend verstanden wird. ${ }^{56}$ Denn viele der modernen Weltraumanwendungen sind potenziell dual-use-fähig, sie haben einen zivil-militärischen Doppelnutzen. ${ }^{57}$ Anschauliche Beispiele bieten das Satellitennavigationssystem GPS und sein russisches Pendant Glonass, die beide unter militärischer Kontrolle sind. ${ }^{58}$ Mittels weltraumgestützter Navigation ist es möglich, Truppenteile und Kampfmittel zielgenauer einzusetzen. Darüber hinaus navigieren auch Millionen privater und kommerzieller Kraftfahrer tagtäglich mit deren Hilfe. Andersherum umfasst beispielsweise ein ziviles Erdbeobachtungssystem wie das künftige europäische Global Monitoring for Environment and Security (GMES) signifikante militärische Bedeutung, zum Beispiel in puncto Aufklärung, Einsatzplanung und -führung oder Spionage.

Die Europäische Union und die ESA sind hinsichtlich dieser sicherheitspolitischen Dimension unterschiedlich ausgerichtet. Schon in den frühen Dokumenten zur EWP lassen sich zurückhaltende Bekenntnisse der Europäischen Union zu einer militärischen Nutzung von Weltraumanwendungen mit dual-use-Charakter herauslesen. ${ }^{59}$ Diese Einstellung änderte sich nachweislich in den darauffolgenden Jahren. So verstärkte der Rat der Europäischen Union diesen Kurswechsel öffentlichkeitswirksam ab 2004 ausgehend von zwei wegweisenden Dokumenten, dem Bericht „European Space Policy: ESDP and Space“ sowie dem anschließenden Aktionsplan „Draft Initial Road Map““ ${ }^{60}$ Der Rat der Europäischen Union bekräftigte hier nicht nur die wechselseitige Bedeutung der beiden Politikfelder Weltraum und Sicherheit, sondern forderte auch deren stärkere Verbindung im Rahmen der damaligen Europäischen Sicherheits- und Verteidigungspolitik (ESVP). Darüber hinaus verlangte der Rat der Europäischen Union die maximale Nutzung der dual-use-Technologien. ${ }^{61}$ Eine Verbindung der beiden Politikfelder erschien somit nicht nur aus finanziellen Gründen sinnvoll zu sein, denn eine Kooperation auf europäischer Ebene - beispielsweise bei der Europäischen Verteidigungsagentur angesiedelt - kann ebenso die organisatorische Abstimmung bei Forschung und Anschaffung effizienter gestalten. In den folgenden Jahren wurden von Europäischer Kommission und Europäischem Parlament weitere Studien zur Verknüpfung der beiden Bereiche in Auftrag gegeben. ${ }^{62}$ Beide Untersuchungen kritisieren die mangelnde Interoperabilität der verschiedenen sicherheitspolitischen Weltraumsysteme und kommen zum

56 Vgl. zum umfassenden/erweiterten Sicherheitsbegriff u.a.: Christopher Daase: Der erweiterte Sicherheitsbegriff, in: Mir A. Ferdowsi (Hrsg.): Internationale Politik als Überlebensstrategie, München 2009, S. 137-153; Lothar Brock: Der erweiterte Sicherheitsbegriff: Keine Zauberformel für die Begründung ziviler Konfliktbearbeitung, in: Die Friedenswarte 3-4/2004, S. 323-326; Heinz Magenheimer: Comprehensive Security. Zum erweiterten Verständnis von Sicherheit, Wien 2001.

57 Gebhard Geiger: Europas weltraumgestützte Sicherheit, Aufgaben und Probleme der Satellitensysteme Galileo und GMES, Stiftung Wissenschaft und Politik: SWP-Studie, Berlin 2005, S. 11.

58 Vgl. Philippe Henry et al.: The militarization and weaponization of space: Towards a European space deterrent, in: Space Policy 2/2008, S. 61-66, hier S. 61.

59 Europäische Kommission: Weißbuch. Aktionsplan für die Durchführung der europäischen Raumfahrtpolitik, 2003, S. 21.

60 Vgl. Rat der Europäischen Union: European Space Policy: ESDP and Space, 11616/3/04; Rat der Europäischen Union: Draft initial road map for achieving the steps specified in the European Space Policy: "ESDP and Space", 9505/05.

61 Der Rat der Europäischen Union bezieht sich hier auf die Vorschläge der folgenden Studie Instituto Affari Internazionali: Internationaler Bericht über Weltraum- und Sicherheitspolitik in Europa, Rom 2003, vor allem S. 3-6. In diesen Zeitraum fällt ebenso ein WEU-Dokument, in dem ähnlich für eine Verknüpfung von Weltraum und ESVP argumentiert wird: Assembly of Western European Union: The Space Dimension of the ESDP, A/ 1881, 30.11.2004.

62 Vgl. Space and security panel of experts: Report of the Panel of Experts on Space and Security, Brüssel 2005; Europäisches Parlament, Generaldirektion Externe Politikbereiche der Union: The Cost of Non Europe in the Field of Satellite Based Systems, Dezember 2007. 
nachvollziehbaren Schluss, Weltraumanwendungen auf europäischer Ebene anzusiedeln. Anknüpfend an diese Untersuchungen hob das Europäische Parlament 2008 die Vorzüge des Galileo-Projekts auch bei militärischen Einsätzen ausdrücklich hervor. ${ }^{63}$ Generell sprach es sich in dieser Entschließung für eine starke Einbindung von Weltraumprogrammen in die ESVP aus. Trotz differenzierter akademischer Debatten über die Ausrichtung der Union, in denen teilweise vor einer Militarisierung des Weltraums gewarnt wurde, ${ }^{64}$ behält auch die Mitteilung zur EWP von 2007 den eingeschlagenen Kurs bei. ${ }^{65}$

Eine solch deutliche Positionierung für eine militärische Nutzbarmachung der dual-usefähigen Anwendungen hat es von der ESA bisher nicht gegeben und ist derzeit nicht zu erwarten. Sie unterliegt den Zwängen der ESA-Konvention, die sowohl in ihrer Präambel als auch in Art. 2 festhält, dass ihr Auftrag ist, Weltraumpolitik für ausschließlich friedliche Zwecke zu betreiben und zu koordinieren. Es ist allerdings festzustellen, dass sich die ESA sicherheitspolitisch neu ausrichtet und ,friedlich“ eher als ,nicht-aggressiv ${ }^{6}$ denn als ,nichtmilitärisch 'interpretiert. ${ }^{66}$ In den ESA-Agenden 2007 und 2011, die jeweils die Leitlinien für die folgenden Jahre festlegten, lassen sich an zahlreichen Stellen Sicherheitsbezüge nachweisen und inzwischen ist die Integration einer sicherheitspolitischen Dimension in die EWP als Schlüsselaufgabe etabliert. ${ }^{67}$ Trotz dieses vorsichtigen Richtungswechsels, der Synergien etwa in Friedenseinsätzen ermöglichen soll, ist die ESA gegenüber militärischer Weltraumpolitik nach wie vor zurückhaltend eingestellt.

Möchte die Union die sicherheitspolitische Dimension der EWP etwa für die Gemeinsame Außen- und Sicherheitspolitik (GASP) und/oder die GSVP nutzbar machen, wie konstatiert werden kann, könnte die ESA einen bedeutenden Beitrag für die Umsetzung entsprechender Projekte leisten. Durch die unterschiedliche Ausrichtung entsteht jedoch ein Spannungsfeld zwischen beiden Organisationen, welches durch das EU-ESA-RA nicht gewürdigt wird. Die dual-use-Fähigkeit moderner Weltraumanwendungen wird durch den Vertrag nicht berücksichtigt, in dem Sicherheit im Katalog der Kooperationsbereiche ${ }^{68}$ keine Erwähnung findet.

Es wird deutlich, dass am Beispiel der industrie- und sicherheitspolitischen Dimension der EWP Konfliktpotenzial zwischen den Akteuren Europäische Union und ESA besteht. Das EU-ESA-RA behebt diese - neben anderen Kooperationsschwierigkeiten - bis dato nicht.

\section{Impulse der Europäischen Kommission 2011}

Zur Verbesserung der bestehenden Kooperationsgrundlage wurden im letzten Jahrzehnt verschiedene Modelle vornehmlich von Stephan Hobe et. al. ${ }^{69}$ entwickelt und intensiv diskutiert: das Beitritts-, das Integrations- und das Kooperationsmodell. Im Folgenden werden

63 Europäisches Parlament: Entschließung des Europäischen Parlaments vom 10. Juli 2008 zu Weltraum und Sicherheit, in: Amtsblatt der EU, Nr. C 294E vom 3. Dezember 2009, S. 69-75; Europäisches Parlament: Entschließung 41. Bericht von Wogau A6-0250/2008, in: Amtsblatt der EU, Nr. C 256E vom 9. Oktober 2008, S. 329-331. Siehe dazu auch später den Abschnitt „Umstrittene Einsatzmöglichkeiten - eine Frage der Sicherheit?".

64 Vgl. Henry et al.: The militarization and weaponization of space, 2008; Frank Slijper: From Venus to Mars, The European Union's steps towards the militarization of space, Amsterdam 2008; Frank Slijper: The EU should freeze its military ambitions in space, in: Space Policy 2/2009, S. 70-74.

65 Europäische Kommission: Europäische Raumfahrtpolitik, 2007, S. 8.

66 Slijper: The EU should freeze its military ambitions in space, 2009, S. 72.

67 Vgl. European Space Agency: Agenda 2007, 2003; European Space Agency: Agenda 2011, 2007.

68 Art. 3 EU-ESA-RA.

69 Hobe/Kunzmann/Reuter: Rechtliche Rahmenbedingungen, 2006; Hobe/Heinrich/Kerner/Froehlich: Entwicklungen der Europäischen Weltraumagentur, 2009. 
der Stand der Diskussion dargestellt, Vor- und Nachteile der Modelle gegenübergestellt und deren Realisierungswahrscheinlichkeit bewertet.

Abbildung 1: Darstellung der Kooperationsoptionen zwischen EU und ESA

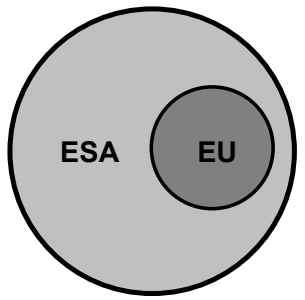

Beitrittsmodell

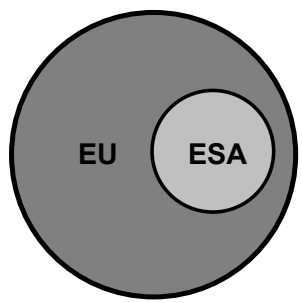

Integrationsmodell

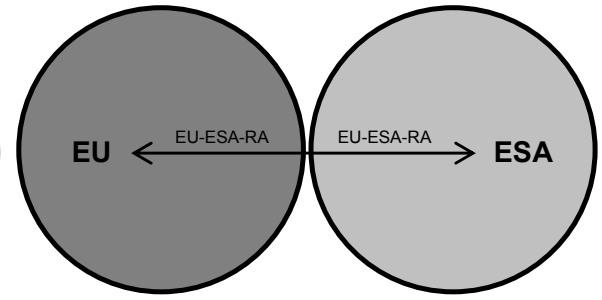

Kooperationsmodell

Quelle: Eigene Darstellung.

Das Beitrittsmodell schlägt vor, dass die Europäische Union der ESA als ordentliches Mitglied beitritt. ${ }^{70}$ Da die Europäische Union im Gegensatz zu den anderen ESA-Mitgliedern kein Staat ist, würde sie eine Sonderstellung im ESA-Gefüge einnehmen. ${ }^{71}$ Zur Verdeutlichung können Parallelen zur Mitgliedschaft der Europäischen Union in der Welthandelsorganisation oder Eurocontrol gezogen werden. ${ }^{72}$ Würde die Europäische Union der ESA beitreten, müssten rechtliche und institutionelle Anpassungen - sowohl auf Seiten der Europäischen Union als auch der ESA - vorgenommen werden. ${ }^{73}$ Gegen die Umsetzung des Beitrittsmodells spricht, dass dieses mit einer Unterordnung der Europäischen Union unter die ESA und damit einem Verlust politischer Gestaltungsmöglichkeiten einherginge. Die Europäische Union bringt maßgeblich politische Leitlinien und Finanzmittel in die EWP ein. Vor diesem Hintergrund scheint es unwahrscheinlich, dass ein Beitrittsmodell, nach dem das weltraum- und wirtschaftspolitische Schwergewicht Europäische Union den gleichen Einfluss wie einzelne ESA-Mitgliedstaaten und nur eine Stimme bei Abstimmungen hätte, politischen Rückhalt unter den EU-Mitgliedstaaten erhielte. ${ }^{74}$ Auch müssten im Vorfeld etliche Sondervereinbarungen zwischen Europäischer Union und ESA getroffen werden, die bestehende Konfliktfelder wie jenes der militärischen Weltraumpolitik oder der Auftragsvergabe ausräumen. ${ }^{75}$

Das Integrationsmodell bildet das theoretische Gegenstück zum Beitrittsmodell. Es umschreibt eine Vielzahl von Ansätzen, um die ESA in die institutionellen Strukturen der Eu-

70 Vgl. Stephan Hobe: Prospects for a European space administration, in: Space Policy 20/2004, S. 25-29, hier S. 27; Hobe/Kunzmann/Reuter: Rechtliche Rahmenbedingungen, 2006, S. 260-281.

71 Vgl. Hobe: Prospects for a European space administration, 2004, S. 27.

72 Vgl. Hobe/Kunzmann/Reuter: Rechtliche Rahmenbedingungen, 2006, S. 262, S. 267-268, S. 269-270; Thomas Hoerber: ESA + EU: Ideology or pragmatic task sharing?, in: Space Policy 4/2009, S. 206-208, hier S. 207; Frans von der Dunk: Towards one captain on the European spaceship - why the EU should join ESA, in: Space Policy 2/2003, S. 83-86, hier S. 85

73 Vgl. ebenda, S. 85-86.

74 Vgl. Hobe: Prospects for a European space administration, 2004, S. 27; Hobe/Kunzmann/Reuter: Rechtliche Rahmenbedingungen, 2006, S. 280.

75 Vgl. ebenda, S. 276, S. 280. 
ropäischen Union zu integrieren. ${ }^{76}$ Denkbar wäre zum Beispiel eine Übernahme des ESATätigkeitsspektrums durch bestehende Organe und Institutionen der Europäischen Union oder gar die Schaffung einer eigenen Weltraumagentur. ${ }^{77}$ Für das Integrationsmodell kann auf die Inkorporation des Tätigkeitsfeldes der Westeuropäischen Union in die Strukturen der Europäischen Union als Muster verwiesen werden. ${ }^{78}$ Voraussetzung für das Integrationsmodell ist stets die Auflösung der ESA an sich. ${ }^{79}$ Das Integrationsmodell ginge folglich mit einer Unterordnung der ESA gegenüber der Europäischen Union einher. Gegen das Integrationsmodell spricht, dass es die jahrzehntelange Erfahrung und die Grundprinzipien der ESA unterminieren würde. ${ }^{80}$ Die ESA als erfahrener Akteur der EWP müsste ihre eigene Auflösung beschließen, was politisch kaum denkbar ist. Somit verwundert es nicht, dass die ESA sich gegen das Integrationsmodell ausspricht. ${ }^{81}$ Weiterhin wäre je nach Ausgestaltung der ESA-Integration eine Änderung des Primärrechts der Europäischen Union mit dem entsprechenden Ratifikationsverfahren erforderlich. ${ }^{82}$

Das Kooperationsmodell bildet am ehesten den Status quo ab. Zugleich ist das Modell derart flexibel, dass es ebenfalls in weiterentwickelter Form als zukünftige Kooperationsgrundlage zwischen Europäischer Union und ESA diskutiert wird. Das Modell geht davon aus, dass die Europäische Union und die ESA weiterhin unabhängig agieren und ausschließlich mittels eines völkerrechtlichen Vertrags miteinander verbunden sind. ${ }^{83}$ Ausgehend von der mäßigen Tragkraft des EU-ESA-RA wird das Kooperationsmodell stetig weiterentwickelt. Ein oberflächlicher Vorteil des Kooperationsmodells liegt darin, dass es beiden Organisationen ermöglicht, auf ihren untereinander unvereinbaren Strukturen und Prinzipien zu beharren. ${ }^{84}$ Eine Weiterentwicklung der EU-ESA-Zusammenarbeit auf Basis weiterer völkerrechtlicher Verträge oder eines überarbeiteten EU-ESA-RA ist denkbar und eröffnet vielfältige Möglichkeiten, ${ }^{85}$ die dem Auftrag im Art. 189 AEUV gerecht würden. Status quo der Diskussion ist der Vorschlag, das Kooperationsmodell zu einem Konstrukt der ESA als Umsetzungsagentur der EWP auf Grundlage eines verbesserten EU-ESA-RA zu optimieren. Der Ansatz, die ESA als ,implementing agency ${ }^{686}$ zu etablieren geht davon aus, dass bisher auf Grundlage des EU-ESA-RA faktisch ohnehin lediglich Ad-hoc-Kooperationen oder EUESA-Zusammenarbeiten denkbar waren, in denen die ESA als Durchführungsagent der Europäischen Union agierte. ${ }^{87}$ Diese Erfahrung könne durch eine Fokussierung auf das ESAManagement von Weltraumanwendungen der Europäischen Union nutzbar gemacht werden.

Hier setzen jüngste Überlegungen der Europäischen Kommission an, welche die Zusammenarbeit zwischen der Europäischen Union und der ESA im Zuge eines verstärkten Engagements der Union in der Weltraumpolitik auf eine neue Grundlage stellen will. Die dabei

76 Vgl. Hobe: Prospects for a European space administration, 2004, S. 26; Hobe/Kunzmann/Reuter: Rechtliche Rahmenbedingungen, 2006, S. 303-338; Hoerber: ESA + EU: Ideology or pragmatic task sharing?, 2009, S. 206-207.

77 Vgl. Hobe/Kunzmann/Reuter: Rechtliche Rahmenbedingungen, 2006, S. 317-338.

78 Vgl. Hoerber: ESA + EU: Ideology or pragmatic task sharing?, 2009, S. 207.

79 Vgl. Hobe/Kunzmann/Reuter: Rechtliche Rahmenbedingungen, 2006, S. 10-11, S. 305-312.

80 Vgl. Hobe/Kunzmann/ Reuter: Entwicklungen der Europäischen Weltraumagentur, 2009, S. 10-11, S. 304; Hoerber: ESA + EU: Ideology or pragmatic task sharing?, 2009, S. 206.

81 Vgl. Hobe: Prospects for a European space administration, 2004, S. 27.

82 Vgl. Hobe/Kunzmann/Reuter: Rechtliche Rahmenbedingungen, 2006, S. 267.

83 Vgl. Hobe: Prospects for a European space administration, 2004, S. 26-27; Hobe/Kunzmann/Reuter: Rechtliche Rahmenbedingungen, 2006, S. 282-302; Hoerber: ESA + EU: Ideology or pragmatic task sharing?, 2009, S. 207.

84 Vgl. Hobe: Prospects for a European space administration, 2004, S. 27.

85 Vgl. Hobe/Kunzmann/Reuter: Rechtliche Rahmenbedingungen, 2006, S. 301.

86 Hobe/Heinrich/Kerner/Froehlich: Entwicklungen der Europäischen Weltraumagentur, 2009.

87 Vgl. ebenda, S. 304. 
gewählten Formulierungen sind außergewöhnlich aufschlussreich, wenn die Kommission „die Erfolge und die Expertise, die sich die Mitgliedstaaten und die Europäische Weltraumorganisation (ESA) erarbeitet haben“ als ein „wertvolles Erbe“ bezeichnet, auf das sich die „schrittweise Entwicklung der Zuständigkeit der Europäischen Union für die Weltraumpolitik stützt [...]. “88 Hier wird der Vorschlag, die Aufgaben der ESA der Europäischen Kommission zu übertragen, kaum verschleiert. Die Europäische Kommission geht aber noch einen Schritt weiter, wenn sie eine Reform der ESA geradezu fordert: „Das zunehmende Engagement der Europäischen Union in der Weltraumpolitik erfordert eine Neubewertung ihrer Beziehungen zur ESA und eine schrittweise Anpassung der Funktionsweise der ESA [...]. “89 Sie stellt richtig fest, dass momentan innerhalb der ESA Überlegungen zur zukünftigen Gestaltung stattfinden. Wie mögliche Reformen aussehen könnten, schlägt die Europäische Kommission dabei auch gleich vor. ${ }^{90}$ Die ESA soll die Organisationsformen schaffen, in denen militärische und zivile Projekte umgesetzt werden können. Des Weiteren wird angeregt, die Annäherung an die Europäische Union fortzusetzen und Strukturen zu schaffen, in denen ausschließlich Unionsprogramme umgesetzt werden. „Diese Organisationsform sollte so flexibel sein, dass sie an die Finanzmittel angepasst werden kann, die die Akteure für die verschiedenen künftigen Programme bereitstellen." ${ }^{\text {"91 }}$ Somit soll sich die ESA bei Projekten mit Beteiligung der Europäischen Union den Finanzierungsmodalitäten und dem Vergaberecht der Union unterwerfen.

Die von der Europäischen Kommission vorgeschlagenen Reformen würden eine einschneidende Neuorientierung für die ESA bedeuten. Die Europäische Union wäre künftig Auftraggeber gegenüber der ESA als Auftragnehmerin. Die ESA, die bisher nur zivile Weltraumprojekte entwickelt hat, müsste sich - nach Änderung der ESA-Konvention - bereiterklären, militärische Programme für die Europäische Union umzusetzen. Die Finanzierung laufe ebenfalls nach Vorgaben der Europäischen Union ab. All dies hätte in jedem Fall eine Neufassung des EU-ESA-RA zur Folge. Inwieweit sich die ESA-Mitgliedstaaten auf eine derart weitreichende Reform und Annäherung an die Europäische Union einlassen, bleibt derzeit abzuwarten.

\section{Das Flaggschiffprojekt Galileo im Spannungsfeld der Interessengegensätze zwischen ESA und EU}

Satellitennavigation ist unbestritten eine der wichtigsten Weltraumanwendungen. Die zivilen Anwendungen und militärischen Einsätze sind ausgesprochen vielfältig und von besonders großer Bedeutung, sodass sich auch die Europäische Union, die ESA und deren jeweilige Mitgliedstaaten schon seit Beginn der 1990er Jahre um die Errichtung eines Satellitennavigationssystems bemühten. Das derzeitige Ergebnis dieser Bemühungen - Galileo - wird seit den Anfängen von Experten aus Politik, Wirtschaft und Wissenschaft kontrovers diskutiert. Eine zentrale und in diesem Zusammenhang immer wieder gestellte Frage in der Debatte lautet: „Braucht Europa ein Satellitennavigationssystem?“992

Galileo ist das älteste umfangreiche Projekt, an dem die Europäische Union und die ESA zusammenarbeiten und kann daher als ein Testfall für die weitere Kooperation der beiden

88 Europäische Kommission: Auf dem Weg zu einer Weltraumstrategie, 2011, S. 3.

89 Ebenda, S. 13.

90 Ebenda.

91 Ebenda.

92 Europäische Kommission: Galileo am Scheideweg: Die Umsetzung der Europäischen GNSS-Programme, 2007, S. 7. 
Organisationen gesehen werden. Aus der Entwicklung und Umsetzung können im Folgenden Schlüsse auf die institutionelle Kooperation dieser beiden Organisationen gezogen werden. ${ }^{93}$

\section{Projektspezifische Ablauforganisation - eine Frage der Finanzen?}

Der Rat der Europäischen Union entschied 2007 unter anderem über die Finanzierung des Galileo-Projekts, das - nach längeren kontroversen Diskussionen - nunmehr ausschließlich aus Haushaltsmitteln der Europäischen Union finanziert wird. ${ }^{94}$ Zudem wurde 2007 eine klarere Rollenverteilung geschaffen, was bedeutet: Rat der Europäischen Union und Europäisches Parlament sind gemeinsame Haushaltsbehörde und wichtigste Gremien bei politischen Entscheidungen bezüglich der Galileo-Programme. Des Weiteren heißt es in den Beratungsergebnissen zu Verwaltung und Ausführung: ,[...] die Kommission sollte die globale Zuständigkeit für die Gesamtverwaltung der Programme als Programmverwalter des europäischen GNSS haben, während die Mitgliedstaaten eng in die zentralen Elemente der Programmverwaltung einbezogen werden sollten. “95

Der ESA wurde die Verantwortung für die Aufbauphase übertragen. Auch sollte die ESA die Auftragsvergabe in dieser Phase übernehmen und sich dabei weitestgehend an entsprechende Regeln und Wettbewerbskriterien der Europäischen Union - und damit nicht an das ESA-Rückflussprinzip - halten. ${ }^{96}$ Für die Sicherheitsakkreditierung des Systems sei die GSA zuständig. ${ }^{97}$ „Wenn der Betrieb des Systems die Sicherheit der EU oder ihrer Mitgliedstaaten gemäß der Gemeinsamen Aktion 2004/552/GASP berührt, sollten die Verfahren der Gemeinsamen Aktion befolgt werden." $" 98$

Durch diese Ratsentscheidung wurden mehrere der aufgetretenen Probleme entschärft, die ein Scheitern des Galileo-Projekts zu diesem Zeitpunkt nicht unwahrscheinlich erscheinen ließen. Mit der vollständigen Finanzierung aus dem EU-Haushalt ${ }^{99}$ und ohne die notwendige Einigung auf eine Public-Private-Partnership wurde die vermutlich größte Hürde überwunden. Die Rollenverteilung zwischen ESA und Europäischer Union wird mit dieser Ratsentscheidung etwas eindeutiger, denn die ESA sollte jetzt die Aufträge nach Vergaberegeln der Europäischen Union und im Einklang mit dem EU-ESA-RA erteilen. Die Europäische Union ist Auftraggeberin und die ESA Auftragnehmerin. Ob und inwieweit die Vergaberegeln angesichts der Struktur der europäischen Raumfahrtindustrie eingehalten werden, bleibt eine offene Frage. ${ }^{100}$ Ebenso offen bleibt auch ein Ende der Diskussion um eine mögliche Kommerzialisierung, die beispielsweise nach der Aufbau- oder in der Betriebs-

93 Vgl. Hobe/Kunzmann/Reuter: Rechtliche Rahmenbedingungen, 2006, S. 138.

94 Vgl. Rat der Europäischen Union: Beratungsergebnisse: Mitteilung der Kommission an das Europäische Parlament und den Rat - GALILEO: Die Europäischen GNSS-Programme mit neuem Profil, 16090/07; vgl. Hobe/Heinrich/Kerner/Froehlich: Entwicklung der Europäischen Weltraumagentur, 2009, S. 94-95.

95 Rat der Europäischen Union: GALILEO: Die Europäischen GNSS-Programme mit neuem Profil, 2007, S. 4. Das Global Navigation Satellite System (GNSS) ist eine satellitengestützte Anwendung zur weltweiten Positionsbestimmung und Navigation. Die Galileo Supervisory Authority (GSA) ist eine EU-Agentur, die in Abstimmung mit der Europäischen Kommission für den Aufbau und die Funktionsfähigkeit von Galileo zuständig ist.

96 Vgl. ebenda, S. 5.

97 Vgl. ebenda.

98 Ebenda; dort heißt es weiter: „Die Aufsicht und die Integration der Sicherheitsanforderungen in das Gesamtprogramm sollten durch den Ausschuss für die europäischen GNSS-Programme gewährleistet werden“.

99 Dadurch wurde die Gemeinschaft auch alleiniger Eigentümer von Galileo und EGNOS. Vgl. ebenda, S. 6.

100 Vgl. zum Vergabeverfahren: Hobe/Heinrich/Kerner/Froehlich: Entwicklung der Europäischen Weltraumagentur, 2006, S. 194-245; Europäische Kommission: Bericht der Kommission an das Europäische Parlament und den Rat. Halbzeitüberprüfung der europäischen Satellitennavigationsprogramme, KOM (2011) 5, S. 3-6. 
phase umgesetzt werden könnte. Einige Bereiche des Projektablaufs sind nach wie vor ungeklärt.

\section{Umstrittene Einsatzmöglichkeiten - eine Frage der Sicherheit?}

Das Satellitennavigationssystem Galileo soll fünf verschiedene Dienste anbieten: ${ }^{101} \mathrm{Zu}$ nächst ist der Open Service als ein kostenloser Basisdienst zu nennen, der dem Empfänger Position, Geschwindigkeit sowie Zeit liefert. Zusatzinformationen zu diesem Basisdienst bietet der kostenpflichtige Commercial Service durch zwei zusätzliche Signale, um eine höhere Genauigkeit zu erreichen. Der sicherheitskritische Safety of Life Service liefert nochmals verbesserte Daten und ein garantiertes Signal mit erhöhter Integrität. Ein Search and Rescue Service soll Notsignalsender verstärken und die Anwendung für Rettungsdienste verbessern. Mit dem Public Regulated Service (PRS) wird ein verschlüsseltes Signal mit sehr hoher Genauigkeit, Qualität und Zuverlässigkeit für staatliche Anwendungen zur Verfügung gestellt, zum Beispiel für Polizei, Küstenwache oder Geheimdienste.

Angesichts dieser Bandbreite an Diensten sind die Einsatzmöglichkeiten von Galileo im Bereich der Sicherheitspolitik umfangreich - und ebenso kontrovers. ${ }^{102}$ Die Anwendungen können vielfältig für boden-, see- und luftgestützte Fahrzeuge genutzt werden. In militärischen GSVP-Operationen würde der PRS die verbesserte Koordination von militärischen Einheiten und Nachschubwegen sicherstellen, Truppenbewegungen sollten mittels der optimierten Synchronisation beschleunigt werden. Militärische Einsätze würden mit satellitengestützter Navigation in jedem Fall besser durchgeführt werden als mit klassischen Navigationssystemen. ${ }^{103}$ Eine weitere Einsatzmöglichkeit des PRS ist die Lenkung von ferngesteuerten Waffen ins anvisierte Ziel, deren Treffgenauigkeit signifikant erhöht werden kann. Bei Operationen im Rahmen der GSVP im oberen Intensitätsspektrum der PetersbergAufgaben, die eines Tages unabhängig von der NATO ${ }^{104}$ und ohne Beteiligung der USA durchgeführt werden sollen, kann ein eigenständiges europäisches Satellitennavigationssystem somit von besonderem militärischen Nutzen sein. ${ }^{105}$

101 Vgl. zu den fünf Diensten Galileos: Susanne Donner: Das europäische Satellitennavigationssystem GALILEO - wirtschaftliche Bedeutung, historische Entwicklung und aktueller Stand, Wissenschaftliche Dienste des Bundestags 57/2005, Berlin 2005; Andreas Mager: Galileo und GPS, Hochtechnologie im Weltraum, in: Strategie und Technik 7/2005, S. 16-19; Geiger: Europas weltraumgestützte Sicherheit, 2005, S. 14-15; Jan Wendel: Integrierte Navigationssysteme. Sensordatenfusion, GPS und Inertiale Navigation, München 2007, S. 111-115; Dickow: Die Weltraumpolitik der EU, 2011, S. 14-15.

102 Vgl. Académie de Marine, Bureau de Longitudes, Académie Nationale de l'Air et de l'Espace (Hrsg.): A positioning system Galileo. Strategic, scientific and technical stakes, Toulouse 2005; Massimo Annati: GALILEO vs. GPS. Battle over Navigation Warfare?, in: Military Technology 12/2003, S. 10-19; Martin W. Buchenau: Ein Navigator für Galileo, in: Handelsblatt, 2.11.2004; Dickow: Die Weltraumpolitik der EU, 2011; Dinerman: China and Galileo continued, 2006; Bernd Eissfeller/Torben Schüler: Das Europäische Satellitennavigationssystem GALILEO, in: GPS und GALILEO. Methoden, Lösungen und neueste Entwicklungen, Beiträge zum DVW-Seminar am 21./22. Februar in Darmstadt, DVW-Schriftenreihe, Augsburg 2006, S. 17-39; Geiger: Europas weltraumgestützte Sicherheit, 2005; Susanne Härpfer: Satellitenkrieg dank Galileo, in: Blätter für deutsche und internationale Politik 12/2006, S. 1427-1429; Ajey Lele: Galileo: EU's footprint in space, in: Indian Defence Review 2/2004, S. 28-36; Europäischer Wirtschafts- und Sozialausschuss: Stellungnahme zu der „Mitteilung der Kommission an den Rat, das Europäische Parlament, den Europäischen Wirtschafts- und Sozialausschuss und den Ausschuss der Regionen - Galileo am Scheideweg: die Umsetzung der europäischen GNSS-Programme“, in: Amtsblatt der EU, Nr. C 191 vom 17. August 2007, S. 17-21.

103 Vgl. Thomas Beer: Die Rolle des Weltraums in der Europäischen Sicherheits- und Verteidigungspolitik, in: Europäische Sicherheit 1/2007, S. 23-30, hier besonders S. 27; Geiger: Europas weltraumgestützte Sicherheit, 2005, S. 17.

104 North Atlantic Treaty Organization.

105 Vgl. Lindström/Gasparini: The Galileo satellite system and its security implications, 2003, S. 21-22. 
Die verschiedenen Auslegungen des Sicherheitsbegriffs, die nicht mehr sinnvoll zu trennenden Zusammenhänge zwischen ziviler und militärischer Sicherheit sowie die ungeklärten Fragen zu dual-use-Problemen initiierten eine kontroverse Debatte, die bis heute andauert. Die dabei vertretenen Meinungen reichen von der konsequenten Streichung bis zur bestmöglichen militärischen Nutzung des Galileo-Projekts. Vertreter der Europäischen Kommission und der ESA haben immer wieder klar zum Ausdruck gebracht, dass Galileo ein „ziviles System unter ziviler Kontrolle“106 sei. Dennoch ist seit einigen Jahren zunehmend festzustellen, dass militärische Nutzungen von Galileo nicht mehr konsequent abgelehnt werden. ${ }^{107}$

Die potenzielle Relevanz von Galileo im Bereich der Verteidigungspolitik wird von mehreren sicherheitspolitischen Experten bestätigt. „There is little doubt, however, that the desire/perceived need to break the US monopoly for security reasons has always been the dominating element, although this was rarely mentioned openly." ${ }^{108}$ Geiger konstatiert: „Insgesamt haben die internationalen Kontroversen um Galileo und GMES eine Reihe sicherheitspolitischer Probleme aufgedeckt, die nicht einfach mit der EU-Parole von den ,zivilen Projekten unter ziviler Kontrolle' zu erledigen sind: Verschränkung ziviler und militärischer Nutzungsarten der Weltraumtechnik mit weitreichenden sicherheitspolitischen Konsequenzen [...].“"109

Die Befürwortung einer sicherheits- und verteidigungspolitischen Nutzung wurde durch eine Entschließung des Europäischen Parlaments unterstützt, die im Juli 2008 mit 483 gegen 99 Stimmen bei 20 Enthaltungen angenommen wurde. ${ }^{110}$ Angesichts der neuen sicherheitspolitischen Bedrohungen und Herausforderungen sei eine verteidigungspolitische Nutzung von Weltraumanwendungen sinnvoll. „Das Europäische Parlament betont, dass Galileo für eigenständige ESVP-Operationen notwendig ist, wie auch für die Gemeinsame Außen- und Sicherheitspolitik, für Europas eigene Sicherheit und für die strategische Autonomie der Union, und gibt zu bedenken, dass insbesondere sein staatlich regulierter Dienst im Bereich Navigation, Ortung und Zeitbestimmung von ausschlaggebender Bedeutung sein wird, nicht zuletzt, um unnötige Risiken zu vermeiden." ${ }^{111}$

Eine Einbindung der ESA, die gemäß ihrer Konvention keine militärischen Anwendungen umsetzen und betreiben darf, ist bei diesen Überlegungen zu sicherheits- und verteidigungspolitischen Nutzungen bislang in ausreichendem Maße vorgenommen worden. Auch wenn noch keine endgültige Entscheidung über die Galileo-Einsatzmöglichkeiten gefallen ist, sollte diese nicht so lange hinausgezögert werden, bis die Einflussmöglichkeiten der ESA nach den ersten Entwicklungsphasen abnehmen.

106 Europäische Kommission: Halbzeitüberprüfung der europäischen Satellitennavigation, 2011, S. 15. So auch die Formulierung der deutschen Bundesregierung: „Galileo garantiert daneben aber eine eigenständige Nutzung. Obwohl es ein ziviles System unter ziviler Kontrolle ist, setzt Galileo auch auf Verfügbarkeit im Krisenfall.“, Deutsche Bundesregierung: Galileo, abrufbar unter: http:/www.bundesregierung.de/Content/DE/ Magazine/MagazinInfrastrukturNeueLaender/017/t4-galileo-phase-zwei-beginnt.html (letzter Zugriff: 16.1.2012).

107 Vgl. Härpfer: Satellitenkrieg dank Galileo, 2006, S. 1427.

108 Annati: GALILEO vs. GPS, 2003, S. 11; vgl auch Lele: Galileo: EU's footprint in space, 2004.

109 Geiger: Europas weltraumgestützte Sicherheit, 2005, S. 12.

110 Europäisches Parlament: Entschließung zu Weltraum und Sicherheit, S. 69-75; Europäisches Parlament: Entschließung 41. Bericht von Wogau A6-0250/2008, in: Amtsblatt der EU, Nr. C 256E vom 9. Oktober 2008, S. 329-331.

111 Europäisches Parlament: Entschließung zu Weltraum und Sicherheit, 2008, S. 72. 


\section{Fazit: Hürden auf dem Weg zu einer kohärenten europäischen Weltraumpolitik}

Nach den ersten politischen Ansätzen zu einer kohärenteren EWP in den 2000er Jahren ergibt sich aus Art. 189 AEUV nunmehr auch eine rechtliche Verpflichtung für die Europäische Union, eine EWP auszuarbeiten und zweckdienliche Verbindungen zur ESA herzustellen. Somit besteht zwangsläufig ein Handlungsbedarf seitens der Union.

Weltraumpolitik kann kaum sinnvoll getrennt von anderen Politikbereichen betrachtet werden, was sich immer wieder als besondere Herausforderung, aber auch ausdrückliche Chance herausstellt. Der Querschnittscharakter zu verschiedenen weiteren Politikfeldern, auf denen die Union aktiv ist, kann zwar zu verstärkten Abstimmungsproblemen führen, allerdings ebenso für einzigartige Synergieeffekte genutzt werden.

Die Analyse der einschlägigen Literatur sowie von EU- und ESA-Dokumenten hat ergeben, dass sich Interessenkonflikte häufig auf zwei Themenfelder konzentrieren, die sich auch bei Galileo deutlich gezeigt haben: Finanzen und Sicherheit. Die Finanzierung von per se kostspieligen Weltraumanwendungen spielt gerade in Zeiten knapper Kassen und schrumpfender Haushalte eine besondere Rolle bei der Erarbeitung und Umsetzung eines gemeinsamen europäischen Weltraumprogramms. In Sicherheitsfragen ist eine kontinuierliche Annäherung zwischen Europäischer Union und ESA zu erkennen, die noch von einer vollständigen Einigung - beispielsweise auf eine gemeinsame Weltraumsicherheitsstrategie - recht weit entfernt scheint. Ein derartiges Übereinkommen könnte allerdings den Grundstein für eine Ausweitung der Kooperation auf Bereiche der GASP legen, wie es im Interesse der sicherheitspolitisch engagierten EU-Mitgliedstaaten ist. ${ }^{112}$

In der Gesamtbetrachtung lässt sich eine Annäherung der beiden Hauptakteure der EWP, nicht zuletzt anhand des gemeinsamen Flaggschiffprojekts Galileo, feststellen. Im Zuge dieser verbesserten politischen Abstimmung ist die weitergehende rechtliche Verpflichtung zur Kooperation als ein weiterer Meilenstein zu sehen. Die neuesten Impulse der Europäischen Kommission unterstützen diesen eingeschlagenen Weg, somit gewinnt die dritte weltraumpolitische Ebene, namentlich die europäische Ebene, zunehmend an Bedeutung gegenüber den nationalen und internationalen Ebenen. Als nächster großer weltraumpolitischer Schritt ist eine Neuauflage des EU-ESA-RA zu empfehlen und zu erwarten. Dieses muss - zumindest für die nächste EU-Finanzplanung bis 2020 - eine tragfähige Basis schaffen.

112 Vgl. Markus Hesse: Europäische Weltraumpolitik - Sicherheitspolitische Aspekte, Berlin 2012. 